\title{
Incorporação de Pó de Pneu em uma Formulação para Banda de Rodagem de Pneu de Motocicleta
}

\author{
Ângela A. Gujel \\ Rinaldi S. A. Indústria de Pneumáticos, RS \\ Rosmary N. Brandalise \\ Departamento de Engenharia Química, CCET-UCS \\ Marcelo Giovanela, Janaina S. Crespo \\ Departamento de Física e Química, CCET-UCS \\ Regina C. R. Nunes \\ Instituto de Macromoléculas Professora Eloisa Mano, UFRJ
}

\begin{abstract}
Resumo: A reciclagem de elastômeros vulcanizados tem sido um problema para as empresas geradoras de resíduos elastoméricos, e ao mesmo tempo um amplo campo de estudo para pesquisadores da área. Diante da possibilidade de recuperação desses resíduos, deu-se início a este trabalho, relacionado à incorporação de pó de pneu de automóvel (STR-r) como uma carga, em uma formulação típica para banda de rodagem de pneu de motocicleta (formulação base - FB). O estudo teve início com a caracterização do STR-r, com base na análise granulométrica, análise termogravimétrica (TG) e calorimetria exploratória diferencial (DSC). A incorporação do STR-r na FB foi realizada em misturador aberto, nas proporções de 5 a 20 phr. Foram calculados os parâmetros reométricos de vulcanização e determinadas as propriedades mecânicas como resiliência, dureza (Shore A), resistência à abrasão, à tração e ao rasgamento dos compósitos preparados. Os resultados foram comparados aos da FB de um processo produtivo de uma empresa de pneus para motocicleta. A partir da análise dos resultados dos diferentes compósitos, foi escolhida a formulação com 10 phr de STR-r para ser produzida em escala industrial, ou seja, em misturador fechado, tipo Banbury. Os cálculos do custo de produção foram realizados com o objetivo de avaliar a viabilidade econômica deste desenvolvimento.
\end{abstract}

Palavras-chave: Pó de pneu, propriedades mecânicas, reciclagem.

\section{Incorporation of Solid Rubber Tire Powder into a Formulation for Motorcycle Tire Tread}

Abstract: The recycling of vulcanized elastomers represents a problem for companies generating elastomeric residues and has become a wide field of study for researchers in this area. This study addresses the possible use of these residues and investigates an alternative for their recovery through incorporation of tread powder of cars tires (STR-r) as a filler into a typical formulation for motorcycle tire tread (base formulation - BF). The study began with the characterization of the STR-r, based on granulometric, thermogravimetric (TGA) and differential scanning calorimetry (DSC) analyses. The incorporation of STR-r into BF was carried out in an open mixer, in the proportions of 5 to $20 \mathrm{phr}$. The rheometric parameters of the vulcanization and the mechanical properties of the composites prepared, such as elongation at break, hardness (Shore A), abrasion resistance, tensile and tear strength, were determined. The results were compared with those of BF used in the production process of a motorcycle tire company. Following the analysis of the results for the different composites, the formulation with 10 phr of STR-r was selected to be produced on an industrial scale, that is, in a closed Banbury-type mixer. The calculation of the production cost was carried out in order to evaluate the economic viability of this recycling option.

Keywords: Solid rubber tire powder, mechanical properties, recycling.

\section{Introdução}

Em função de propriedades tais como elasticidade, flexibilidade, impermeabilidade, resistência à abrasão e à corrosão, os materiais elastoméricos são amplamente utilizados nas mais diferentes aplicações ${ }^{[1]}$. Contudo, durante o processo produtivo de artefatos elastoméricos em geral estima-se que seja gerado o índice de $10 \%$ de resíduos ${ }^{[2]}$, quantidade que se torna significativa quando o consumo, no ano de 2006 no Brasil, foi de 400 mil toneladas de borracha sintética e 314 mil toneladas de borracha natural ${ }^{[3]}$. Para a indústria de pneumáticos estima-se que os resíduos gerados no processo produtivo estão na faixa de 1-2\%. No Rio Grande do Sul, a indústria da borracha gera, anualmente,

Autor para correspondência: Janaina S. Crespo, Grupo de Materiais Elastoméricos, Departamento de Física e Química, Centro de Ciências Exatas e Tecnologia, Universidade de Caxias do Sul, Rua Francisco Getúlio Vargas, 1130, CEP: 95070-560, Caxias do Sul, RS, Brasil. E-mail: jscrespo@ucs.br 
cerca de 11.800 toneladas de resíduos, sendo que $87 \%$ correspondem a resíduos industriais classificados segundo NBR 10004-0 $4^{[4]}$ como Classe II - não perigosos e por este motivo devem ter destino adequado ${ }^{[5]}$.

Além dos resíduos gerados no processo produtivo dos artefatos existe ainda o descarte relacionado aos artefatos pós-consumo. Dentro desse contexto, a maior fonte geradora de resíduos são os pneus inservíveis, que contêm em sua composição principalmente o copolímero de butadieno e estireno (SBR) e a borracha natural (NR). A reciclagem de pneus pós-consumo permite minimizar o acúmulo deste resíduo que pode trazer sérios riscos à saúde pública e ao meio ambiente se descartado indevidamente, além de evitar a ocupação de espaço físico, considerando as elevadas proporções geradas ${ }^{[6]}$.

A caracterização do resíduo é primordial quando a incorporação ocorre em composições do processo produtivo, pois pode-se buscar um desempenho técnico semelhante à formulação virgem (sem resíduo), mantendo-se propriedades de interesse tecnológico com a possibilidade de redução dos custos da formulação ${ }^{[7,8]}$.

O objetivo deste trabalho foi realizar um estudo comparativo entre as propriedades da banda de rodagem de um pneu para motocicleta com e sem pó de pneu (STR-r) incorporado, buscando a máxima incorporação, manutenção das propriedades de interesse tecnológico e a possibilidade de redução do custo de produção.

\section{Experimental}

\section{Caracterização do STR-r}

Para o desenvolvimento desse trabalho foi utilizado resíduo de pó de pneu de automóvel (STR-r) proveniente da empresa Borrachas Planalto Ltda, Bento Gonçalves - RS. A caracterização do STR-r foi feita pelo tamanho da partícula, análise termogravimétrica (TG) e calorimetria exploratória diferencial (DSC).

A determinação do tamanho do pó do STR-r foi feita seguindo a norma ASTM D 5644 - $01^{[9]}$, e utilizando peneiras padrão de 24, 28, 35, 48, 65 e 100 mesh.

A determinação das temperaturas de transição vítrea $(\mathrm{Tg})$ dos elastômeros constituintes do STR-r, bem como a identificação do sistema de aceleração residual foi feita por calorimetria exploratória diferencial (DSC) em um equipamento da marca Shimadzu DSC 50 (Kyoto, Japão). As condições de ensaio foram as seguintes: massa de amostra de aproximadamente $10 \mathrm{mg}$, fluxo de nitrogênio de $50 \mathrm{~mL} \cdot \mathrm{min}^{-1}$, em uma faixa de temperatura de -100 a $250{ }^{\circ} \mathrm{C}$, sob duas taxas de aquecimento, 10 e $30{ }^{\circ} \mathrm{C} / \mathrm{min}$. A taxa de aquecimento mais lenta foi utilizada na determinação da $\mathrm{Tg}$, uma vez que esta implica em alta resolução e melhor separação de eventos. A taxa de aquecimento mais rápida, por outro lado, foi utilizada na determinação da vulcanização residual, pois implica em alta sensibilidade e detecção de pequenas transições ${ }^{[17]}$.

A composição parcial do STR-r foi determinada por análise termogravimétrica (TG) em um equipamento Shimadzu TGA-50 (Kyoto, Japão) com fluxo de nitrogênio de $50 \mathrm{~mL} \cdot \mathrm{min}^{-1}$, na faixa de temperatura de 20 a $800{ }^{\circ} \mathrm{C}$ e com taxa de aquecimento de $10{ }^{\circ} \mathrm{C} / \mathrm{min}$, sendo de aproximadamente $10 \mathrm{mg}$ a massa de amostra. Para fins comparativos, o SBR 1502 (Petroflex Indústria e Comércio S/A) foi analisado nas mesmas condições.

\section{Preparação da formulação base e incorporação do STR-r}

A FB utilizada neste estudo teve como componentes os seguintes materiais/reagentes: dois tipos de copolímero de butadieno-estireno (SBR 1502 e SBR 1712 - Petroflex Indústria e Comércio S/A); negro de fumo 339 (Degussa); óleo aromático (Chevron Brasil Ltda); 1,2-dihidro-2,2,4-quinolina polimerizada (Bann Química Ltda); óxido de alumínio silicoso hidratado $\left(\mathrm{Al}_{2} \mathrm{O}_{3} \cdot 2 \mathrm{SiO}_{2} \cdot 2 \mathrm{H}_{2} \mathrm{O}\right.$ - Inducol Indústria de Calcários Caçapava); ácido esteárico (SIM Estearina Indústria e Comércio); óxido de Zinco (ZnO - Votorantin/Metais); parafina (Isogama Indústria Química Ltda); N-(1,3-dimetilbutil)N'-fenil-p-fenilenodiamina (6PPD - Flexsys Indústria e Comércio Ltda); N-cicloexil-2-benzotiazol sulfenamida (CBS Bann Química Ltda); enxofre ventilado (S - RCN \& Phelps Distribuidora Química Ltda); dissulfeto de 2,2-dibenzotiazol (MBTS) e dissulfeto de tetrametiltiuram (TMTD) (Bann Química Ltda).

A FB foi preparada em um misturador fechado do tipo Banbury, modelo MIR 100, (Rio Grande do Sul, Brasil) com capacidade de $100 \mathrm{~L}$. A mistura foi realizada a $190 \pm 10{ }^{\circ} \mathrm{C}$, em um tempo definido de 3,5 minutos, com uma pressão de ar no pilão de $600.000 \mathrm{~Pa}$. A temperatura da água de refrigeração do equipamento foi de $22{ }^{\circ} \mathrm{C}$.

Em seguida, a FB foi conduzida a um misturador aberto de cilindros, modelo MAC 1500, capacidade $90 \mathrm{~kg}$, (Rio Grande do Sul, Brasil), a fim de ser laminada a $100 \pm 10{ }^{\circ} \mathrm{C}$, sendo posteriormente colocada em uma esteira de refrigeração, onde permaneceu por aproximadamente 3,5 minutos. Os aceleradores foram adicionados à $\mathrm{FB}$ em um misturador aberto de cilindros a $100 \pm 10{ }^{\circ} \mathrm{C}$, sendo 3 minutos o tempo de mistura. No final dessa etapa, a FB ficou descansando por 4 horas até sua liberação para utilização.

A incorporação de quantidades variadas de STR-r à FB foi efetuada em um misturador aberto de cilindros de laboratório EEMCO, modelo $\mathrm{LAB}$, capacidade 1,5 kg, (Erie, USA), segundo norma ASTM D 3182-06 ${ }^{[10]}$, na temperatura de $60{ }^{\circ} \mathrm{C}$, sendo a massa total da formulação base de $1,5 \mathrm{~kg}$ e o tempo de mistura de 4 minutos. A adição do STR-r foi realizada nas proporções de 5, 10, 15 e $20 \mathrm{phr}$. 


\section{Determinação dos parâmetros de vulcanização (reômetro de disco oscilatório)}

A FB e os quatro compósitos desenvolvidos com STR-r $(5,10,15$ e $20 \mathrm{phr})$ foram analisados em reômetro de disco oscilatório (ODR) Tech Pro, modelo Rheotech OD+ (Ohio, USA), com freqüência de $1,67 \mathrm{~Hz}$, amplitude de deformação de $1^{\circ}$ e temperatura de $160{ }^{\circ} \mathrm{C}$, conforme norma ASTM D $2084-06^{[11]}$.

\section{Vulcanização e obtenção dos corpos de prova}

Os corpos de prova para a caracterização da FB e dos quatro compósitos com STR-r foram extraídos de placas vulcanizadas, moldadas por compressão em prensa hidráulica, da marca Luxor, (São Paulo, Brasil), a $160{ }^{\circ} \mathrm{C}$, sob pressão de $10 \mathrm{MPa}$, a partir dos valores do tempo ótimo de vulcanização $\left(\mathrm{t}_{90}\right)$, pré-determinados no ODR, mais o acréscimo de um minuto para cada milímetro de espessura, no caso dos corpos de prova para resiliência, resistência à abrasão e dureza (Shore A). Esse procedimento seguiu a norma ASTM D $3182-06^{[10]}$.

\section{Caracterização da FB e dos compósitos desenvolvidos}

Os diferentes compósitos foram caracterizados quanto às propriedades mecânicas, segundo normas específicas para os ensaios propostos.

Os testes de dureza, tração e rasgamento foram realizados antes e após envelhecimento acelerado, em estufa com circulação forçada de ar Erzinger, modelo 90/65/70, (Santa Catarina, Brasil), durante 72 horas a $70{ }^{\circ} \mathrm{C}$ (ASTM D 573-04 $4^{[12]}$ ).

A determinação da resistência à tração e ao rasgamento, antes e após envelhecimento acelerado, foi feita em máquina Universal de Ensaios Emic, modelo DL-3000, (Paraná, Brasil) com velocidade de separação das garras de $500 \mathrm{~mm} / \mathrm{min}$, segundo norma ASTM D 412-06a ${ }^{[13]}$ e ASTM D 624-00 $0^{[14]}$, respectivamente. Para a resistência à tração, os seguintes parâmetros foram avaliados: tensão na ruptura (MPa), alongamento na ruptura (\%) e módulo a 300\% (MPa).

Para a determinação da dureza, segundo norma ASTM D 2240-05 ${ }^{[15]}$, foi utilizado um durômetro Shore A, Teclock, (São Paulo, Brasil). No ensaio de abrasão, em abrasímetro Veb Thüringer, modelo AGPi (Rauenstein, Alemanha), foi calculada a perda de massa do corpo de prova, a partir de uma trajetória de fricção de $40 \mathrm{~m}$ sobre uma lixa de referência. A resistência à abrasão foi expressa em volume de massa perdido em $40 \mathrm{~m}$, ou seja, $\mathrm{mm}^{3} .40 \mathrm{~m}^{-1}$. O ensaio seguiu a norma DIN 53516-8 $7^{[18]}$. O ensaio de resiliência foi realizado em resiliômetro Veb Tiw (Rauenstein, Alemanha) provido de um martelo Charpy, segundo norma ASTM D 1054-02 $2^{[16]}$.

Os parâmetros considerados essenciais do ponto de vista tecnológico para a escolha da formulação com adição de STR-r foram: resistências à tração, à abrasão e ao rasgamento. Após a análise de todos os resultados obtidos para os compósitos com diferentes teores de STR-r incorporados, a composição que atendeu aos requisitos essenciais para uma banda de rodagem, foi a formulação com 10 phr de STR-r.
Esta formulação foi produzida em escala industrial, no misturador fechado do tipo Banbury (mesmas condições da FB), sendo em seguida re-analisada.

\section{Resultados e Discussão}

\section{Caracterização do STR-r}

Na caracterização granulométrica do STR-r, o tamanho médio de partículas ficou entre 48 a 100 mesh $(0,147$ a 0,295 mm). De acordo com a literatura ${ }^{[8,19,20]}$, o tamanho ideal de pó reciclado para a incorporação em novas formulações deve ser menor que $0,6 \mathrm{~mm}$, portanto o STR-r apresentou dimensão adequada para a aplicação proposta.

O STR-r foi analisado também por calorimetria exploratória diferencial (DSC), na determinação das transições vítreas $(\mathrm{Tg})$ dos elastômeros presentes no resíduo, bem como na identificação do sistema de aceleração residual. Os resultados obtidos (Figura 1a) indicam, pela transição vítrea, a presença dos dois elastômeros: a borracha natural, NR, com a Tg em $-57,6{ }^{\circ} \mathrm{C}$, e o copolímero de butadieno-estireno, SBR com a $\mathrm{Tg}$ a $-32,3{ }^{\circ} \mathrm{C}^{[21-24]}$. A presença de sistema de aceleração residual pôde ser confirmada pelo pico exotérmico a $233,6^{\circ} \mathrm{C}$ (Figura $1 \mathrm{~b}$ ), que desapareceu em uma segunda corrida (Figura 1c), e que corresponde ao excesso destes materiais presentes no STR-r.

Foi confirmada pela análise da curva derivada (DTG) a existência de dois elastômeros no STR-r. A DTG apresentou dois estágios de degradação (Figura 2). O primeiro em $377{ }^{\circ} \mathrm{C}$, com perda de massa de $33,0 \%$; e o segundo estágio em $445^{\circ} \mathrm{C}$, com perda de massa de $25,4 \%$, que estão relacionados, respectivamente, à degradação da NR (temperatura de degradação de $380^{\circ} \mathrm{C}^{[25]}$ ) e a do SBR (curva não apresentada, temperatura de degradação de $473{ }^{\circ} \mathrm{C}$ ). $\mathrm{O}$ resíduo de $41,6 \%$ corresponde aos demais aditivos presentes no STR-r, predominantemente ao negro de fumo.

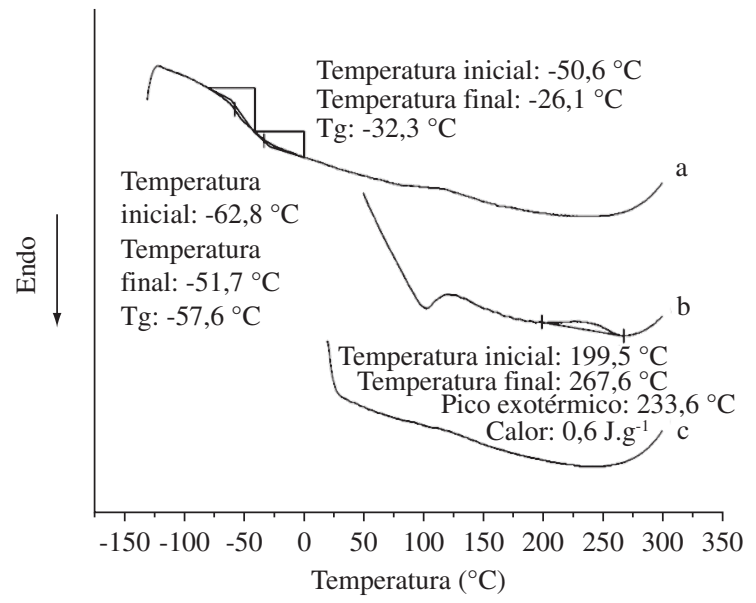

Figura 1. Curvas de DSC do STR-r; a) taxa de aquecimento de $10^{\circ} \mathrm{C} / \mathrm{min}$; b) taxa de aquecimento de $30^{\circ} \mathrm{C} / \mathrm{min}$ (primeira corrida); e c) taxa de aquecimento de $30^{\circ} \mathrm{C} / \mathrm{min}$ (segunda corrida); (fluxo de $\mathrm{N}_{2}$ de $50 \mathrm{~mL} / \mathrm{min}$ e massa de amostra de $10 \mathrm{mg}$ ). 


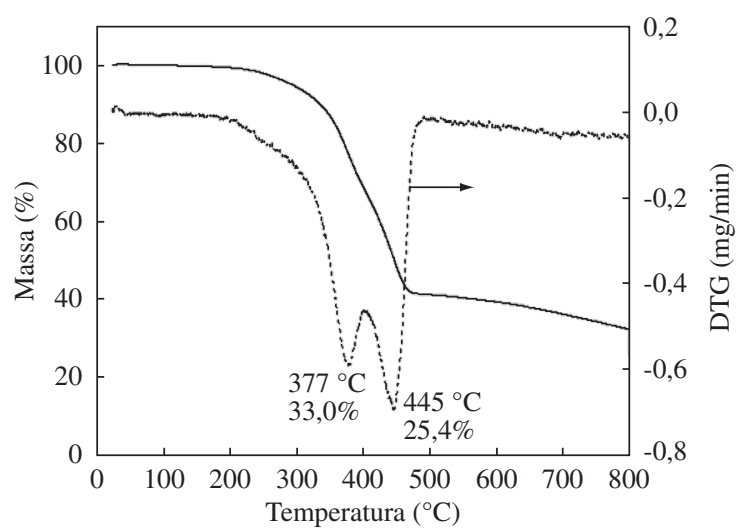

Figura 2. Curvas de TG e DTG do STR-r; (fluxo de $\mathrm{N}_{2}$ de $50 \mathrm{~mL} / \mathrm{min}$, taxa de aquecimento de $10^{\circ} \mathrm{C} / \mathrm{min}$, massa de amostra de $10 \mathrm{mg}$ ).

\section{Caracterização da FB e dos compósitos desenvolvidos}

Os parâmetros reométricos para a FB e para os quatro compósitos desenvolvidos estão apresentados na Tabela 1.

Os resultados obtidos indicam que, com o acréscimo do teor de STR-r, os valores de torque máximo $\left(\mathrm{M}_{\mathrm{H}}\right)$ decrescem, apontando uma menor rigidez molecular, que pode estar relacionada à diminuição do teor de borracha virgem, onde as ligações cruzadas se formam. Os valores de torque mínimo $\left(\mathrm{M}_{\mathrm{L}}\right)$ também diminuem com a incorporação do resíduo, indicando uma menor viscosidade do material, o que é um dado interessante quanto à processabilidade do compósito. A redução na viscosidade do material pode ser atribuída à substituição de parte da matriz elastomérica pelo STR-r que, de acordo com os resultados da análise de TG, apresentou somente $58,4 \%$ de elastômero. A influência da adição do STR-r pode ser avaliada quanto à diferença entre os torques $\Delta \mathrm{M}=\mathrm{M}_{\mathrm{H}}-\mathrm{M}_{\mathrm{L}}$. Em comparação com a FB, os maiores valores de $\Delta \mathrm{M}$ foram encontrados para os compósitos com incorporação de 5 e 10 phr de STR-r, assinalando a contribuição mais efetiva deste resíduo.

O tempo a $90 \%$ de cura $\left(\mathrm{t}_{90}\right)$ é um parâmetro importante, pois indica o tempo ótimo de vulcanização dos compósitos, sem que ocorra degradação e envelhecimento das misturas. O $\mathrm{t}_{90}$ para todas as composições desenvolvidas é menor do que o da FB, com exceção da composição com 10 phr, cujo valor é semelhante ao da FB.

$\mathrm{O}_{\mathrm{S} 1}$ é um parâmetro que indica o tempo de segurança do processo relacionado ao início da formação de ligações cruzadas. As misturas desenvolvidas com a incorporação de

Tabela 1. Parâmetros reométricos de vulcanização da FB e dos compósitos desenvolvidos com diferentes teores de STR-r.

\begin{tabular}{ccccccc}
\hline $\begin{array}{c}\text { Teor de } \\
\text { STR-r } \\
(\mathbf{p h r})\end{array}$ & $\begin{array}{c}\mathbf{M}_{\mathbf{L}} \\
(\mathbf{d N} . \mathbf{m})\end{array}$ & $\begin{array}{c}\mathbf{M}_{\mathbf{H}} \\
(\mathbf{d N . m})\end{array}$ & $\begin{array}{c}\Delta \mathbf{M} \\
(\mathbf{d N} . \mathbf{m})\end{array}$ & $\begin{array}{c}\mathbf{t}_{\mathbf{s 1}} \\
(\mathbf{m i n})\end{array}$ & $\begin{array}{c}\mathbf{t}_{\mathbf{9 0}} \\
(\mathbf{m i n})\end{array}$ & $\begin{array}{c}\mathbf{C R I} \\
\left(\mathbf{m i n}^{-1}\right)\end{array}$ \\
\hline (FB) 0 & 27,7 & 119,3 & 91,6 & 1,51 & 6,03 & 22,12 \\
5 & 26,8 & 124,3 & 97,5 & 2,04 & 4,29 & 44,44 \\
10 & 25,0 & 116,0 & 91,0 & 4,06 & 6,26 & 45,45 \\
15 & 22,7 & 73,1 & 50,4 & 1,35 & 3,43 & 48,07 \\
20 & 16,8 & 59,6 & 42,8 & 2,07 & 3,43 & 73,52 \\
\hline
\end{tabular}

STR-r apresentaram $t_{\mathrm{S} 1}$ similar à formulação base $(0 \mathrm{phr})$, com exceção da mistura com 10 phr de STR-r, que apresentou um tempo de segurança superior ao da FB.

Com base nos resultados de $\mathrm{t}_{90} \mathrm{et}_{\mathrm{s} 1}$, outro parâmetro que pode ser calculado a partir da curva reométrica é o CRI, que é o índice de velocidade de cura $\left[100 /\left(\mathrm{t}_{90}-\mathrm{t}_{\mathrm{s} 1}\right)\right]^{[11]}$. Analisando-se os resultados da Tabela 1, pode-se concluir que o CRI aumenta com o teor de STR-r incorporado, e que, em comparação à $\mathrm{FB}$, todos os compósitos desenvolvidos têm maior velocidade de cura. Esses valores podem ser justificados pelos resultados de DSC obtidos para o STR-r, e já discutidos, que comprovam a presença de sistema de cura residual no STR-r.

Algumas propriedades mecânicas escolhidas para a avaliação da FB e para os compósitos desenvolvidos, como dureza, resiliência, e resistência ao rasgamento e à abrasão, estão mostradas na Tabela 2. Os resultados encontrados para os compósitos desenvolvidos são bastante promissores por apresentarem valores próximos aos da FB. As composições com 5 e 10 phr de STR-r têm melhor desempenho quanto a resistência à abrasão do que a FB, sendo esta propriedade determinante para a escolha de formulação para pneumáticos.

$\mathrm{Na}$ Figura 3 estão mostrados os resultados de módulo a $300 \%$, tensão e alongamento na ruptura para a FB e demais formulações com diferentes teores de STR-r. Os resultados de tração permitem concluir que, de uma maneira geral, é possível a incorporação de até 20 phr de STR-r em uma formulação para banda de rodagem para pneus de motocicleta. Esta afirmativa pode ser justificada pelos resultados obtidos para os compósitos desenvolvidos, e que se encontram dentro do desvio-padrão da FB. Estes resultados são devido não somente ao tamanho da partícula do STR-r, mas também ao tipo de resíduo e às condições de processamento usadas.

Um resultado de interesse tecnológico é a resposta de formulações submetidas ao envelhecimento acelerado. Este experimento foi realizado em estufa com circulação forçada de ar por 72 horas a $70{ }^{\circ} \mathrm{C}$ com todos os compósitos, os quais foram posteriormente ensaiados quanto à dureza, tração e rasgamento. A retenção das propriedades após envelhecimento acelerado, foi calculada considerando-se como $100 \%$ os resultados das propriedades obtidos para os compósitos não envelhecidos (Tabela 2 e Figura 3). Os resultados de retenção da propriedade estão mostrados na Figura 4. A FB apresenta excelente resistência ao envelhecimento nas condições expe-

Tabela 2. Propriedades mecânicas da FB e dos compósitos desenvolvidos com diferentes teores de STR-r.

\begin{tabular}{|c|c|c|c|c|}
\hline $\begin{array}{c}\text { Teor de } \\
\text { STR-r } \\
(\text { phr })\end{array}$ & $\begin{array}{c}\text { Dureza } \\
\text { (Shore A) }\end{array}$ & $\begin{array}{c}\text { Perda por } \\
\text { abrasão } \\
\left(\mathrm{mm}^{3} .40 \mathrm{~m}^{-1}\right)\end{array}$ & $\begin{array}{c}\text { Resiliência } \\
(\%)\end{array}$ & $\begin{array}{l}\text { Resistência } \\
\text { ao } \\
\text { rasgamento } \\
\left(\mathbf{k N} \cdot \mathbf{m}^{-1}\right)\end{array}$ \\
\hline (FB) 0 & $62 \pm 1$ & $165 \pm 8$ & $21 \pm 1$ & $38 \pm 3$ \\
\hline 5 & $58 \pm 3$ & $143 \pm 5$ & $21 \pm 2$ & $36 \pm 1$ \\
\hline 10 & $62 \pm 1$ & $145 \pm 4$ & $20 \pm 2$ & $38 \pm 2$ \\
\hline 15 & $62 \pm 1$ & $187 \pm 7$ & $21 \pm 1$ & $38 \pm 4$ \\
\hline 20 & $60 \pm 1$ & $190 \pm 5$ & $21 \pm 1$ & $36 \pm 3$ \\
\hline
\end{tabular}


rimentais usadas e observa-se que os valores das propriedades das composições com 5, 10, 15 e 20 phr de resíduo são próximos aos da composição sem resíduo.

Uma das definições para carga de reforço está relacionada com seu desempenho quanto às propriedades mecânicas, como resistências à tração, ao rasgamento e à abrasão ${ }^{[26]}$. No caso deste trabalho, pelos resultados obtidos para cada uma dessas propriedades antes e após envelhecimento, o STR-r pode ser classificado como uma carga semi-reforçadora.

Com base nos resultados fornecidos pelas composições desenvolvidas neste trabalho, a mistura com $10 \mathrm{phr}$ de STR-r foi escolhida para ser preparada em escala industrial, visando a sua utilização em uma formulação para banda de rodagem para pneu de motocicleta.

Observando-se os resultados obtidos (Tabela 3 ) verificouse que as propriedades avaliadas para o compósito onde a incorporação de $10 \mathrm{phr}$ de STR-r foi realizada em misturador

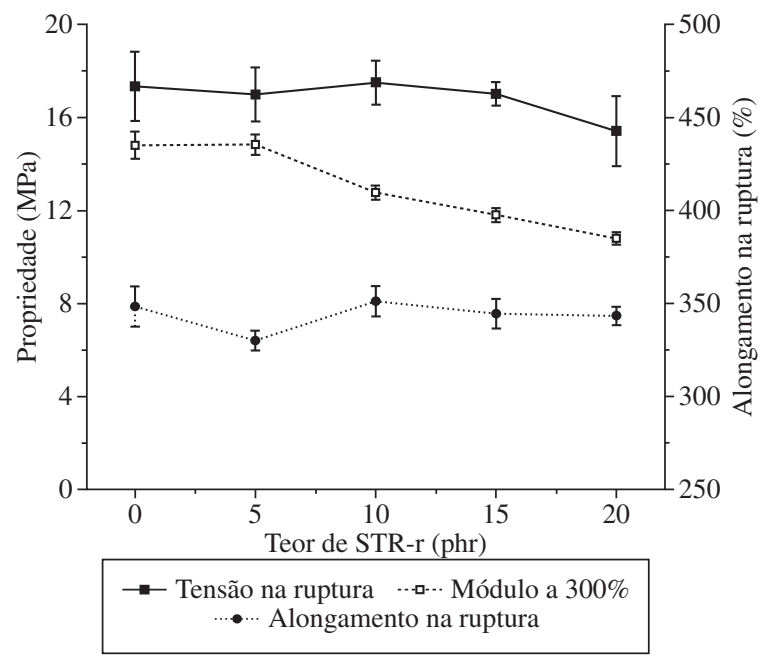

Figura 3. Resultados de resistência à tração da FB e dos compósitos desenvolvidos com diferentes teores de STR-r.

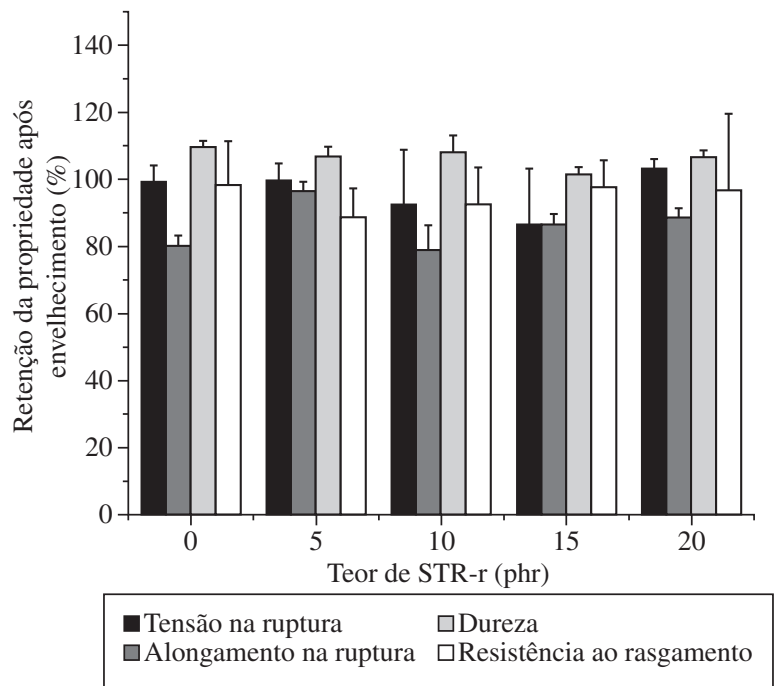

Figura 4. Retenção das propriedades da FB e dos compósitos desenvolvidos com diferentes teores de STR-r após envelhecimento acelerado (72 horas a $\left.70{ }^{\circ} \mathrm{C}\right)$.
Tabela 3. Comparação das propriedades mecânicas da FB e dos compósitos com $10 \mathrm{phr}$ de STR-r preparados em escala laboratorial e escala industrial.

\begin{tabular}{|c|c|c|c|}
\hline \multirow[t]{2}{*}{ Ensaio } & \multicolumn{3}{|c|}{ Teor de STR-r (phr) } \\
\hline & $10^{\mathrm{a}}$ & $10^{b}$ & $\begin{array}{c}\text { Formulação } \\
\text { base }^{c}\end{array}$ \\
\hline $\begin{array}{l}\text { Tensão na ruptura } \\
(\mathrm{MPa})\end{array}$ & $17 \pm 1$ & $15 \pm 1$ & $17 \pm 1$ \\
\hline $\begin{array}{l}\text { Alongamento na } \\
\text { ruptura }(\%)\end{array}$ & $351 \pm 8$ & $316 \pm 13$ & $348 \pm 30$ \\
\hline Dureza (Shore A) & $62 \pm 1$ & $61 \pm 1$ & $62 \pm 1$ \\
\hline $\begin{array}{l}\text { Desgaste por abrasão } \\
\qquad\left(\mathrm{mm}^{3} .40 \mathrm{~m}^{-1}\right)\end{array}$ & $145 \pm 4$ & $164 \pm 7$ & $165 \pm 8$ \\
\hline Resiliência (\%) & $20 \pm 2$ & $18 \pm 2$ & $21 \pm 1$ \\
\hline $\begin{array}{c}\text { Resistência ao } \\
\text { rasgamento }\left(\mathrm{kN} \cdot \mathrm{m}^{-1}\right)\end{array}$ & $38 \pm 2$ & $35 \pm 2$ & $38 \pm 3$ \\
\hline
\end{tabular}

${ }^{\mathrm{a} F o r m u l a c ̧ a ̃ o ~ b a s e ~ c o m ~ i n c o r p o r a c ̧ a ̃ o ~ d e ~} 10$ phr de STR-r realizada em misturador aberto (escala laboratorial); ${ }^{\text {f }}$ ormulação base com incorporação de $10 \mathrm{phr}$ de STR-r realizada em Banbury (escala industrial); e cformulação base preparada em Banbury (escala industrial).

aberto, foram semelhantes àquelas da FB. No caso do compósito com incorporação de $10 \mathrm{phr}$ de STR-r, realizada em Banbury, os resultados obtidos (considerando-se o desviopadrão) encontram-se no limite inferior em relação às propriedades da FB. Esse resultado pode ser justificado devido à diferença de temperatura de incorporação que no Banbury foi de $190{ }^{\circ} \mathrm{C}$ e no misturador aberto de $60^{\circ} \mathrm{C}$. A alta temperatura do Banbury provocou o envelhecimento deste compósito, o que implicou em menor desempenho mecânico.

O custo por quilograma de formulação desenvolvida com $(\mathrm{R} \$ 2,92 / \mathrm{kg})$ e sem $(\mathrm{R} \$ 3,02 / \mathrm{kg}) 10 \mathrm{phr}$ de STR-r $(\mathrm{R} \$ 1,47 / \mathrm{kg})$ foi calculado com base na composição das formulações e no custo de cada matéria-prima. Em seguida, realizou-se uma estimativa mensal de produção destas formulações, considerando-se que as etapas de mistura, processamento e moldagem dos artefatos foram realizadas nas mesmas condições experimentais. A incorporação de 10 phr de STR-r em uma formulação para banda de rodagem para pneu de motocicleta implicou em uma redução de 3,3\% no valor de produção mensal do artefato.

\section{Conclusões}

O pó de pneu utilizado (STR-r) apresentou características adequadas para a aplicação proposta. Em comparação à FB, os compósitos desenvolvidos foram obtidos em menor tempo, pela presença de sistema de aceleração residual no STR-r, conforme observado na análise de DSC. A incorporação de $10 \mathrm{phr}$ de STR-r em uma FB para banda de rodagem para pneu de motocicleta implicou em melhores propriedades mecânicas. A análise das propriedades mecânicas, antes e após envelhecimento acelerado, permitiu a classificação do STR-r como uma carga semi-reforçadora. A incorporação de $10 \mathrm{phr}$ de STR-r em uma formulação para banda de rodagem para pneu de motocicleta implicou em uma redução de 3,3\% no valor de produção mensal do artefato. 


\section{Agradecimentos}

Os autores agradecem à FAPERGS/Procoredes II (Processo $n^{\circ}$ : 0518831) e ao CNPq (Processo no: 481748/2007-5) pelo auxílio financeiro. A empresa Rinaldi S. A. Indústria de Pneumáticos pela confecção do molde flash.

\section{Referências Bibliográfia}

1. Costa, H. M.; Visconte, L. L. Y.; Nunes, R. C. R. \& Furtado, C. R. G. - Polímeros: Cienc Tecnol, 13, p. 125, (2003).

2. Schnecko, H. - Macromol. Symp., 135, p. 327, (1998).

3. Sindicato das Indústrias de Artefatos de Borracha do Estado do RS (SINBORSUL). "Perfil da Indústria de Artefatos de Borracha do Brasil e do RS, 2007”. Disponível em www.sinborsul.com.br (acesso fevereiro 2008).

4. Associação Brasileira de Normas Técnicas - "Resíduos Sólidos - Classificação ABNT NBR 10004”, (2004).

5. Fundação Estadual de Proteção Ambiental (FEPAM). "Relatório sobre a Geração de Resíduos Sólidos Industriais no Estado do Rio Grande do Sul, 2003”. Disponível em www.fepam.rs.gov.br (acesso fevereiro 2008).

6. Adhikari, B.; De, D. \& Maiti, S. - Prog. Polym. Sci., 25, p. 909, (2000).

7. Zanchet A.; Dal'Acqua, N.; Weber, T.; Crespo, J. S.; Brandalise, R. N. \& Nunes, R. C. R. - Polímeros: Cienc Tecnol, 17, p. 23, (2007).

8. Weber, T.; Zanchet, A.; Brandalise, R. N.; Crespo, J. S. \& Nunes, R. C. R - J. Elastom. Plast., 40, p. 147 (2008).

9. American Society for Testing and Materials. "Standard test method for rubber compounding materials - determination of particle size distribution of recycled vulcanized particulate rubber ASTM D 5644-01", West Conshohocken (2007).

10. American Society for Testing and Materials. "Standard practice for rubber-materials, equipment, and procedures for mixing standard compounds and preparing standard vulcanized sheets ASTM D 3182-06”, West Conshohocken (2007).

11. American Society for Testing and Materials. "Standard test method for rubber property - vulcanization using oscillating disk meter ASTM D 2084-06”, West Conshohocken (2007).

12. American Society for Testing and Materials. "Standard test method for rubber - deterioration in an air oven ASTM D 573-04", West Conshohocken (2007).
13. American Society for Testing and Materials. "Standard test methods for vulcanized rubber and thermoplastic elastomers - tension ASTM D 412-06a", West Conshohocken (2007).

14. American Society for Testing and Materials. "Standard test method for tear strength of conventional vulcanized rubber and thermoplastic elastomers ASTM D 624-00”, West Conshohocken (2007).

15. American Society for Testing and Materials. "Standard test method for rubber property - durometer hardness ASTM D 2240-05”, West Conshohocken (2007).

16. American Society for Testing and Materials. "Standard test method for rubber property - resilience using a Goodyear-Healy rebound pendulum ASTM D 1054-02”, West Conshohocken (2007).

17. Machado, L. D. B. \& Matos, J. R. - "Análise térmica diferencial e calorimetria exploratória diferencial", in: Técnicas de Caracterização de Polímeros, Sebastião V. Canevarolo Jr. (ed), Artliber Editora Ltda., São Paulo (2004).

18. Deutsches Institut Fur Normung - "Testing of rubber and elastomers: Determination of abrasion resistence DIN 53516-87', Alemanha (1987).

19. Gibala, D. \& Hamed, G. R. - Rubber Chem. Technol., 67, p. 636, (1994).

20. Brito, K. J. S.; Pacheco, G. S. \& Lovison, V. M. H. "Metrologia e Ensaios Básicos na Indústria da Borracha”. São Leopoldo: Senai, Centro Tecnológico de Polímeros, 2003.

21. Mendes, L. C. \& Mano, E. B. - "Introdução a Polímeros", Edgard Blücher, São Paulo (1999).

22. Visconte, L. L. Y.; Martins, A. F.; Nunes, R. C. R. \& Suarez, J. - Polimeros: Cienc Tecnol, 11, p.76, (2001).

23. Moreno, R. M. B.; Mattoso, L. H. C.; Job, A. E. \& Gonçalves, P. S. - Polimeros: Cienc Tecnol, 16, p.235, (2006).

24. Kumnuantip, C. \& Sombatsompop, N. - Mater. Lett., 57, p.3167, (2003).

25. Dutra, R. C. L.; Diniz, M. F.; Ribeiro, A. P.; Lourenço, V. L.; Cassu, S. N. \& Azevedo, M. F. P. - Polimeros: Cienc Tecnol, 14, p.334, (2004).

26. Mark, J. E.; Erman, B. \& Eirich, F. R. - "Science and Technology of Rubber", Academic Press, New York (1994).

Enviado: 30/03/08

Reenviado: 05/06/08

Aceito: $11 / 06 / 08$ 